\title{
Nondestructive Imaging of Dielectric-Constant Profiles and Ferroelectric Domains with a Scanning-Tip Microwave Near-Field Microscope
}

\author{
Yalin Lu, Tao Wei, Fred Duewer, Yanqing Lu, \\ Nai-Ben Ming, P. G. Schultz, X.-D. Xiang*
}

Variations in dielectric constant and patterns of microwave loss have been imaged in a yttrium-doped $\mathrm{LiNbO}_{3}$ crystal with periodic ferroelectric domains with the use of a scanning-tip near-field microwave microscope. Periodic profiles of dielectric constant and images of ferroelectric domain boundaries were observed at submicrometer resolution. The combination of these images showed a growth-instability-induced defect of periodic domain structure. Evidence of a lattice-edge dislocation has also been observed through a stress-induced variation in dielectric constant.

Crystals with periodic and quasi-periodic ferroelectric domain structures, such as $\mathrm{LiNbO}_{3}, \mathrm{LiTaO}_{3}$, and $\mathrm{KTiOPO}_{4}$ superlattice crystals $(1,2)$ (either in bulk form or as thin-film waveguides), have attracted considerable interest and found important applications in quasi-phase matched nonlinear optics (3) and in acoustics (4). Currently, a destructive method involving optical imaging of differentially etched surfaces is commonly used to characterize the domain structures. Several other (mainly charge- or polarization-sensitive) techniques have also been developed (5), but none allows nondestructive, high-resolution imaging of ferroelectric domains over a large area. Moreover, there is no effective technique to analyze the variations in dielectric constant corresponding to variations in dopant concentration, which could give rise to domain formation, in these materials and related devices.

We have previously developed a scanning-tip microwave near-field microscope with $5-\mu \mathrm{m}$ resolution (6) and have now improved its performance to nondestructively image surface dielectric constant and microwave loss with submicrometer spatial resolution and a scanning range of over 2.5 $\mathrm{cm}$. Through images of dielectric constant, we have observed submicrometer-resolution profiles of periodic dielectric constant and evidence of a lattice-edge dislocation in a yttrium-doped $\mathrm{LiNbO}_{3}$ superlattice crystal. The profiles of microwave energy loss yield nondestructive images of ferroelectric do-

Y. Lu, F. Duewer, X.-D. Xiang, Molecular Design Institute, Lawrence Berkeley National Laboratory, Berkeley, CA 94720, USA.

T. Wei and P. G. Schultz, Howard Hughes Medical Institute and Molecular Design Institute, Lawrence Berkeley National Laboratory, Berkeley, CA 94720, USA.

Y. Lu and N.-B. Ming, National Laboratory of Solid State Microstructures, NanJing University, NanJing 210093, China.

*To whom correspondence should be addressed. E-mail: xdxiang@|bl.gov main boundaries. By correlating the images of both dielectric constant and microwave energy loss, a growth-instability-induced defect in the periodic domain structure has been identified. These studies should contribute significantly to our understanding of the growth mechanism of the crystals. In addition, the imaging technique used here should prove useful in analyzing other ferroelectric and dielectric materials and thinfilm devices.

The design of our scanning-tip microwave near-field microscope involves a sharpened metal tip mounted on the center conductor of a high-quality factor (high-Q) coaxial resonator that extends beyond an aperture formed in the end wall of the resonator (6). The spatial resolution of the microscope increases with the sharpness of the tip, without the exponential decrease in sensitivity that is a characteristic of hollow wave guides used in conventional near-field microscopes. Increases in microwave energy loss (absorption) and dielectric constant at the surface of the samples near the tip lead to a decrease in $Q$ and resonant frequency $f_{0}$ of the resonator, respectively. By recording $Q$ and $f_{0}$, we can image the surface profiles of dielectric constant and microwave energy loss in the sample.

Yttrium doping has proven to be an effective method of introducing a periodic ferroelectric domain structure (superlattice) in the as-grown $\mathrm{LiNbO}_{3}$ single crystal (7). For this study, an average of 0.5 weight $\%$ yttrium-doped $\mathrm{LiNbO}_{3}$ superlattice single crystal was grown along the $a$ axis ( $x$ axis in Fig. 1 ) with the Czochralski technique at the Nanjing University (7). A 3-mm-thick wafer cut normal to the $y$ axis and polished on both surfaces was used. The surface of the crystal was examined with a profilometer to confirm the optical quality smoothness. In this configuration, the polarizations of the ferroelec- tric domains alternate along the $c$ axis $(z$ axis in Fig. 1). Two domain laminas with opposite polarization give rise to the same surface charge configuration (because there is no $y$-axis component of spontaneous polarization), simplifying the interpretation of our experimental results (chargesensitive techniques fail to image the structures in this configuration). The microscope tip scans over the $x z$ plane of the sample approximately along the $x$ axis (with a typical scan speed of 1 to $5 \mu \mathrm{m} / \mathrm{s}$ in this study). The electrochemically etched tungsten tip (with typical radius of $\sim 0.1$ $\mu \mathrm{m}$ ) was kept in contact with the surface of the sample with a soft spring (with a spring constant of $4 \mathrm{~N} / \mathrm{m}$ and a typical contact force of $<20 \mu \mathrm{N}$ ). No surface damage was observed with this scanning format. Neither the scanning format nor the detected signal is sensitive to topography (that is, material features with identical dielectric constant and tangent loss but different height are not distinguishable in the image).

A periodic variation in the concentration of yttrium was generated by growing the $\mathrm{LiNbO}_{3}$ crystal along the $x$ axis in an asymmetric temperature field $(1,8)$. The periodic variation in dopant concentration induces (through the internal spacecharge field) the formation of ferroelectric domains with alternating polarization when the crystal undergoes a para-to-ferroelectric phase transition. Although alternating polarization does not modulate its second-rank tensor dielectric constant ( $\mathrm{LiNbO}_{3}$ belongs to the $3 \mathrm{~m}$ point group, for which the nonzero second-rank tensor $\varepsilon_{11}, \varepsilon_{22}$, and $\varepsilon_{33}$ does not change its sign under the transformation of $180^{\circ}$ rotation around the $x$ axis), the periodic variation of dopant level should result in a periodic change in the dielectric constant. Therefore, in the Czochralski-grown $\mathrm{LiNbO}_{3}$ superlattice crystals, two periodic structures (that is, dielectric constant and ferroelectric domain) should coexist, although the former has never been observed previously. We have observed both periodic struc-

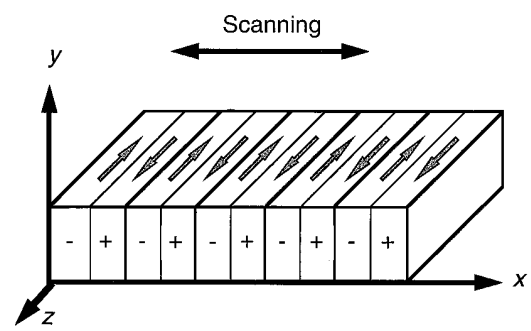

Fig. 1. Schematic of a $\mathrm{LiNbO}_{3}$ crystal containing periodic ferroelectric domains. Polarization is along the optical axis ( $z$ axis), and scanning is along the $x$ axis. 

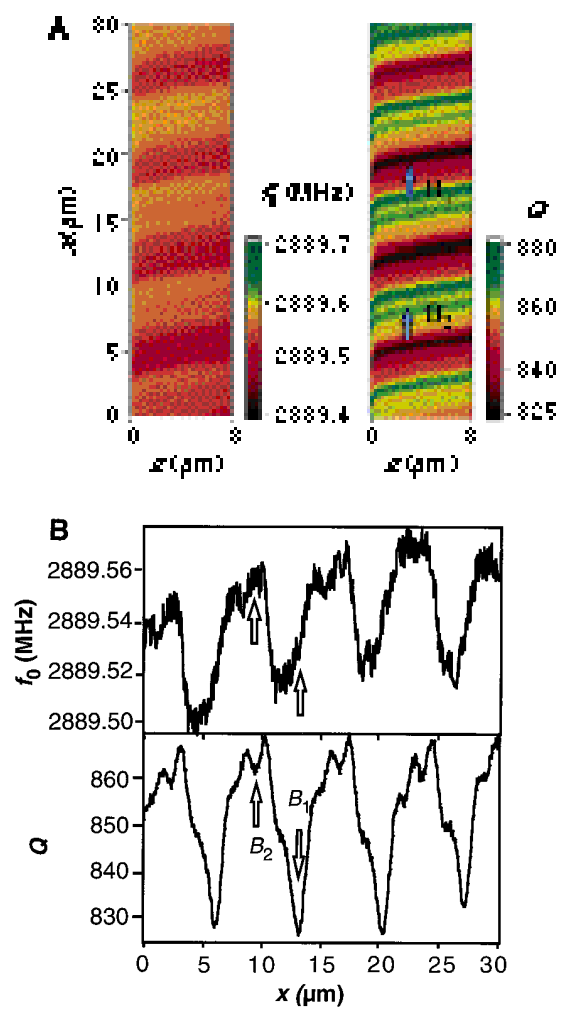

Fig. 2. (A) Images of profiles of periodic dielectric constant and ferroelectric domain boundaries. The $f_{0}$ profile mainly indicates variations in dielectric constant when the change in loss is not large, and the $Q$ profile indicates the microwave energy loss of the sample surface. (B) The $f_{0}$ and $Q$ profiles of (A) along the $x$ axis at $z=4.0 \mu \mathrm{m}$. The diffuse and sharp domain boundaries are marked $\mathrm{B}_{1}$ and $\mathrm{B}_{2}$, respectively.

tures through microwave imaging (Fig. 2). The image of resonant frequency $f_{0}$ reflects variations in the dielectric constant associated with changes in dopant levels, whereas the image of $Q$ corresponds to losses in microwave energy, which are large at the ferroelectric domain boundaries (primarily as the result of movement of the domain walls under the influence of the microwave field). The change in resonant frequency is $\Delta f_{0} / f_{0}=$ $g \Delta \varepsilon^{\prime}$ (9), where $\varepsilon=\varepsilon^{\prime}+i \varepsilon^{\prime \prime}$ is the complex dielectric constant and $g$ was measured to be $\sim 7 \times 10^{-5}$ in this configuration. The total dielectric variation in Fig. 2 is estimated to be $\sim 0.25$ with a noise level of 0.03 , which is the current sensitivity of the microscope. The change in cavity quality factor is $\Delta(1 /$ Q) $=g \Delta \varepsilon^{\prime \prime}(9)$, and $\Delta \varepsilon^{\prime \prime}$ was calculated to be $7.1 \times 10^{-2}$. The total loss tangent variation in Fig. 2 is estimated to be about $1 \times 10^{-2}$. Detailed theoretical analysis and experimental calibration of the sensitivity and accuracy were performed (10).

The domain boundaries with relatively low loss (thin yellow stripe in Fig. 2A marked as $\mathrm{B}_{2}$ ) are located at the dielectric constant minima ( $f_{0}$ maxima in Fig. $\left.2 \mathrm{~B}\right)$,
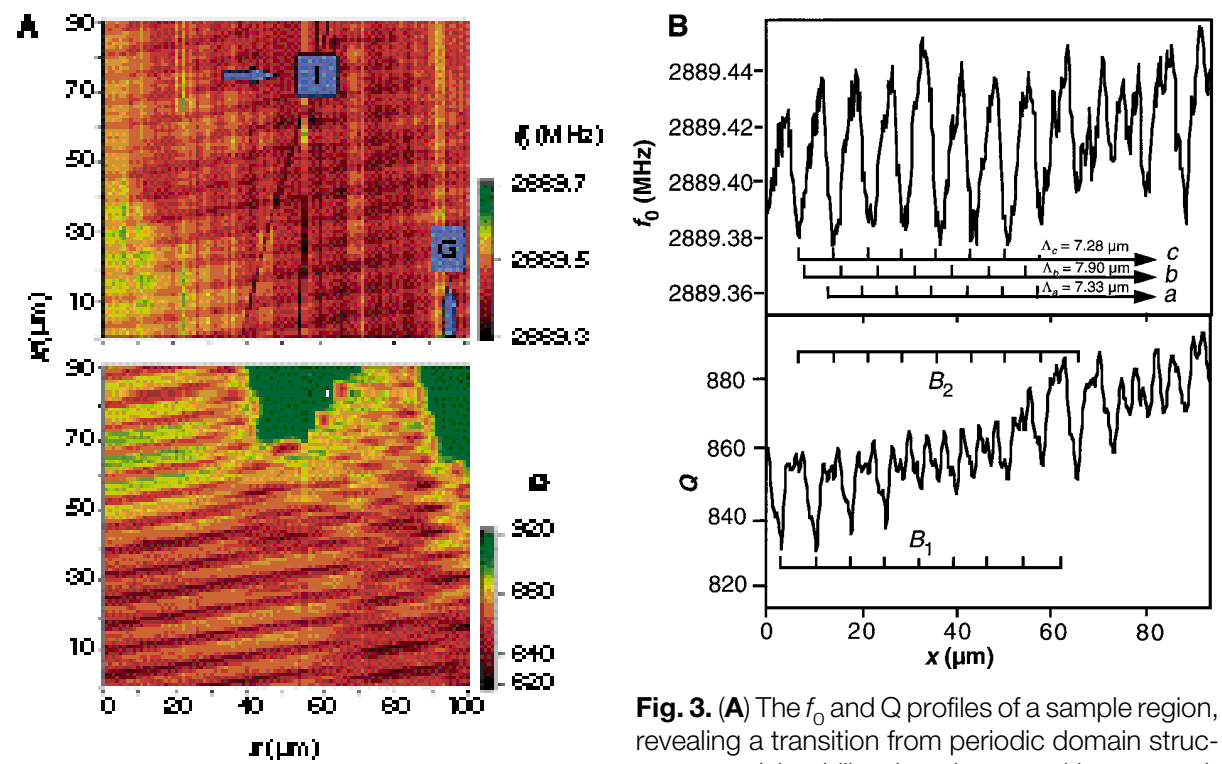

Fig. 3. (A) The $f_{0}$ and $Q$ profiles of a sample region, revealing a transition from periodic domain structure to an island-like domain caused by a growth instability. The island-like domain is marked as "I," and the growth direction, as "G." (B) The $f_{0}$ and Q profiles of (A) along the $x$ axis at $z=73 \mu \mathrm{m}$. In the $f_{\mathrm{O}}$ profile, several modulation frequencies $\Lambda$ (with periods of $7.33,7.90$, and $7.28 \mu \mathrm{m}$ ) are marked $a, b$, and $c$.

whereas the domain boundaries with high loss (wide dark-red stripe in Fig. 2A marked as $\mathrm{B}_{1}$ ) are located at a critical value of the gradient in dopant concentration (where the polarization changes its direction) near (but not at) the dielectric constant maxima $\left(\varepsilon_{\max }\right)$. The full width at half maxima of $B_{1}$ $(\sim 2.5 \mu \mathrm{m})$ is greater than that of the $B_{2}$ $(\sim 1 \mu \mathrm{m})$. This result is consistent with the previous finding of sharp and diffuse boundaries by $\mathrm{x}$-ray energy dispersive spectral analysis in these crystals (11) and the proposed scenario that diffuse domain boundaries do not occur at dopant maxima (12) if we assume that the doping of yttrium in the $\mathrm{LiNbO}_{3}$ crystals monotonically increases the dielectric constant. Because diffuse domain boundaries are not always located at the dopant maxima (corresponding to $\left.\varepsilon_{\max }\right)$, alternating polarization domains separated by domain boundaries (as observed in images of Q) may also have different widths. The width ratio of the two domains in one period of $7.0 \mu \mathrm{m}$ (Fig. 2) is $\sim 0.87$.

Because dopant (dielectric constant) and domain boundary profiles can be imaged simultaneously, it is possible to gain information about the growth mechanism of the crystal by correlating the detailed features of the profiles. For example, we observed a defect in the periodic domain structure (that is, an island-like domain caused by solid-liquid interface instability during growth) ("I" in Fig. 3A). This type of defect is common in Czochralski-grown $\mathrm{LiNbO}_{3}$ crystals and is destructive to the periodic domain structure (12). As is evident from the $f_{0}$ profile, the dopant modulation is more diffuse near the island, and the overall dopant level is higher in the island region. A corresponding transition from sharp (low loss) to diffuse (high loss) domain boundaries is clearly observable in the $Q$ profile. These observations indicate

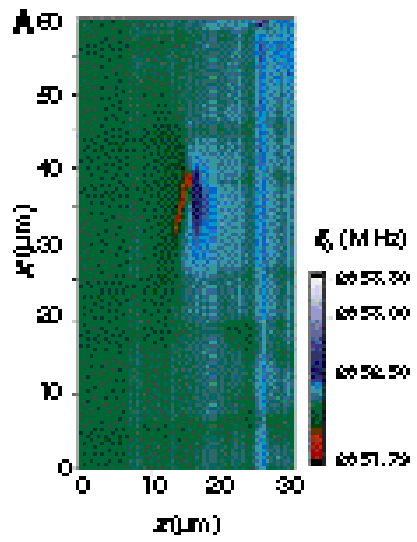

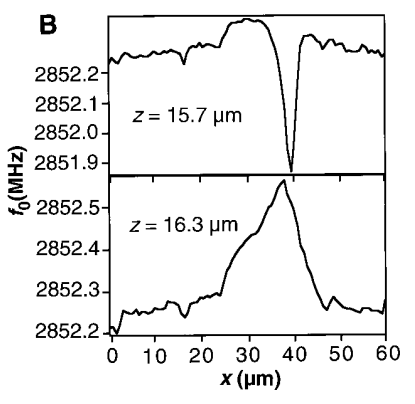

Fig. 4. (A) The $f_{0}$ profiles of a sample region that indicate a lattice dislocation. (B) The $f_{0}$ profile of $(A)$ along the $z$ axis at $x=38.7 \mu \mathrm{m}$. 
that dopant aggregation, caused by temperature instabilities in the island region during the crystal growth, is the origin of the island-like domains. A gradual transition from sharp to diffuse domain boundaries is again evident in $Q$ and $f_{0}$ profiles at $z=73 \mu \mathrm{m}$ (Fig. 3B), and the domain structure gradually loses its original periodicity approaching the island domain. For stable growth, the periodic modulation of dopant level should have a single frequency (which is the frequency of crystal rotation) $(1,8)$, whereas disturbances in the system, such as melt or air convection and power fluctuations, may introduce additional modulations with different frequencies. When these additional frequencies are close to the crystal rotation frequency (with a period of $\sim 7 \mu \mathrm{m}$ in Fig. 3), interference eventually drives the solidliquid interface out of stability, causing dopant aggregation and island domain formation during crystal growth (Fig. 3B).

A "butterfly" image of the dielectric constant is an indication of a lattice "edge dislocation" defect (Fig. 4A). The large difference in dielectric constant adjacent to the dislocation is caused by compressive and tensile stresses in lattice structure induced by the dislocation. The stress-induced $\varepsilon$ contour is qualitatively consistent with the theoretical prediction for a lattice "edge dislocation" defect (13). The $\varepsilon$ profile of a line scan along the $z$ axis at $x=38.7 \mu \mathrm{m}$ (Fig. 4B), which cuts through regions with compressive and tensile stresses, shows large opposite changes in $\varepsilon$ on either side of the edge defect. This profile also indicates that our spatial resolution in this configuration (mainly limited by the radius of the tip) is better than $1 \mu \mathrm{m}$, because between the two points $(0.6 \mu \mathrm{m}$ apart), $\varepsilon$ changed from the minimum to near maximum. Lattice dislocations in $\mathrm{LiNbO}_{3}$ crystals are not observable by optical microscopy with polarized light because of the crystal's large birefringence.

\section{REFERENCES AND NOTES}

1. D. Feng and N. B. Ming, Appl. Phys. Lett. 37, 607 (1980).

2. N. B. Ming et al. , J. Mater. Sci. 17, 1663 (1982); G. A. Magel et al. , Appl. Phys. Lett. 56, 108 (1990); Y. L. Lu and N. B. Ming, ibid. 69, 1660 (1996).

3. J. A. Armstrong and N. Bloembergen, Phys. Rev. 127, 1918 (1962).

4. See, for example, S. D. Cheng, Y. Y. Zhu, Y. L. Lu, N. B. Ming, Appl. Phys. Lett. 66, 291 (1995), and references therein.

5. T. Ozaki et al., J. Appl. Phys. 80, 1697 (1996); R. LeBihan and M. Maussion, J. Phys. 33, C2-215 (1972); P. J. Lin et al., Philos. Mag. A 48, 251 (1983); F. Saurenbach and B. D. Terris, Appl. Phys. Lett. 56, 1703 (1990)

6. T. Wei, X. D. Xiang, P. G. Schultz, Appl. Phys. Lett. 68, 3506 (1996).

7. Y. L. Lu, Y. Q. Lu, N. B. Ming, ibid., p. 2781.

8. Y. L. Lu, L. Mao, N. B. Ming, ibid. 59, 516 (1991).

9. H. A. Bethe and J. Schwinger, Perturbation Theory of Cavities (National Defense Research Committee,
Washington, DC, 1943), pp. D1-117.

10. C. Gao, F. Duewer, X.-D. Xiang, in preparation.

11. J. Chen, Q. Zhou, J. F. Hong, W. S. Wang, D. Feng, J. Appl. Phys. 66, 336 (1989)

12. Y. L. Lu, Y. Q. Lu, N. B. Ming, Appl. Phys. Lett. 68 , 2642 (1996), and references therein.

13. J. P. Hirth and J. Lothe, Theory of Dislocations
(Krieger, Malabar, FL, ed. 2, 1992).

14. This work was supported by the director of Advanced Energy Projects Division, Office of Computational and Technology Research, U.S. Department of Energy, under contract DE-AC03-76SF00098.

\title{
Observation of Magnetic Domain Behavior in Colossal Magnetoresistive Materials With a Magnetic Force Microscope
}

\author{
Qingyou Lu, Chun-Che Chen, Alex de Lozanne*
}

Magnetic domain behavior was imaged in calcium-doped lanthanum manganese trioxide films. Magnetic domains behave differently at temperatures that are well below and near the Curie temperature $T_{\mathrm{c}}$. At low temperatures the domains are very stable. As temperature rises toward $T_{c}$, domain-domain interactions become weaker and domains become highly mobile and subject to merging and splitting, yet the domains are still traceable. The contrast (which is a measure of magnetization) and size of domains continuously reduce to zero. The temperature at which the domains disappear agrees with the $T_{\mathrm{c}}$ obtained by a macroscopic magnetization measurement. Sample defects such as scratches tend to attract magnetic domains.

Metallic double-exchange ferromagnets, such as $\mathrm{LaMnO}_{3}$ doped with doubly valenced impurities, were discovered and studied by Jonker and Van Santen in 1950 (1). Pure $\mathrm{LaMnO}_{3}$ is an antiferromagnetic insulator with a well-known perovskite structure. When a divalent dopant $\mathrm{D}$ (D can be $\mathrm{Ca}, \mathrm{Sr}, \mathrm{Ba}$, or $\mathrm{Pb}$ ) is introduced, the $\mathrm{La}_{1-x} \mathrm{D}_{x} \mathrm{MnO}_{3}$ that is generated exhibits paramagnetism at high temperatures and ferromagnetism at low temperatures for a wide doping range around $x=1 / 3$. This phase transition has been commonly interpreted in terms of the double-exchange interaction model that was first proposed in 1951 (2). The substitution of $\mathrm{La}^{3+}$ by a $\mathrm{D}^{2+}$ cation results in a mixed $\mathrm{Mn}^{3+} / \mathrm{Mn}^{4+}$ valence. The electron can hop from one $\mathrm{Mn}$ ion to another $\mathrm{Mn}$ ion through $\mathrm{O}^{2-}$ in between. Because Hund's rule must be obeyed on $\mathrm{Mn}$ sites, this hopping process can occur only when the ionic spins of two $\mathrm{Mn}$ ions are parallel. The motion of the electrons thus causes a partial alignment of the Mn spins that in turn lowers the kinetic energy and favors the occurrence of ferromagnetism. However, there are other results that do not support this model (3).

Another intriguing feature of doped $\mathrm{LaMnO}_{3}$ is the magnetoresistive effect that was first measured on a flux-grown single

\section{Department of Physics, University of Texas, Austin, TX} 78712-1081, USA.

*To whom correspondence should be addressed. E-mail: lozanne@physics.utexas.edu crystal in 1969 (4). Shortly thereafter, the dependence of resistivity on temperature and the applied magnetic field was obtained by Kubo and Ohata by exploitation of the well-known Drude approximation in terms of a Kondo lattice with ferromagnetic coupling (5).

Although it has been studied for years, the behavior of doped $\mathrm{LaMnO}_{3}$ is far from well understood and is still attracting extensive investigation because of its value in basic research as well as its possible application in magnetic recording. Whereas much attention has recently been paid to bulk magnetization and structural properties (6), we have been trying to understand the behavior of the local magnetization in thin films of this compound at the microscopic level. We used a low-temperature magnetic force microscope (LTMFM) to study the magnetic domain behavior in colossal magnetoresistive $\mathrm{La}_{0.65} \mathrm{Ca}_{0.35} \mathrm{MnO}_{3}$ films.

The $\mathrm{La}_{0.65} \mathrm{Ca}_{0.35} \mathrm{MnO}_{3}$ thin films were grown on $\mathrm{SrTiO}_{3}(100)$ substrates by electron-beam and thermal co-evaporation (7) of the metallic elements in an oxygen partial pressure of $4 \times 10^{-6}$ torr at a substrate temperature of $700^{\circ} \mathrm{C}$. After deposition, ex situ annealing was performed at $960^{\circ} \mathrm{C}$ in air for $30 \mathrm{~min}$. The thickness of the films was $300 \mathrm{~nm}(8)$.

The dependence of sample resistivity $\rho$ and magnetization $\mathrm{M}$ on temperature $T$ in different magnetic fields $H$ applied in the plane of the thin film (Fig. 1) shows the colossal magnetoresistance effect. Without 